\title{
Neurocognitive and Neurobehavioral Effects of Deployment: The Implications of Service during the "War on Terror"
}

\author{
Maxine H. Krengel
}

Received: 23 January 2012 / Accepted: 30 January 2012 /Published online: 15 February 2012

(C) Springer Science+Business Media, LLC 2012

The United States initiated the "War on Terror" over 10 years ago. Hundreds of thousands of troops deployed to Iraq (Operation Iraqi Freedom; OIF) and Afghanistan (Operation Enduring Freedom; OEF) have suffered emotional and physical consequences that have superseded those experienced by individuals deployed to other conflicts. Further, these war-related consequences continue to be incompletely identified, diagnosed, and understood. Although research in the areas of neurocognitive and neuroradiological correlates has increased significantly in the past several years, only now are formal reviews of the literature emerging, such as those presented in this special section of Neuropsychology Review.

These reviews are timely because clinicians are in the process of determining gold standard measures to assess post-deployment cognitive dysfunction, neuroradiological correlates to assist in diagnostic accuracy, and state-of-theart treatments for post-deployment traumatic brain injury and comorbid affective disorders. This set of papers begins with an overview by Bogdanova and Verfaellie on the research to date on the neuropsychological and neuroradiological correlates of symptoms consistent with OEF/OIF deployment. It is the first paper to present a critical literature review of the methodology of published studies and an integration of neuroradiological findings.

Given the large proportion of post-deployment men and women who have comorbid psychiatric and neurocognitive symptoms, Dolan, Martindale, Robinson, Kimbrel, Meyer,

M. H. Krengel $(\bowtie)$

Department of Neurology, Boston University School of Medicine, 725 Albany Street,

Boston, MA 02118, USA

e-mail:mhk@bu.edu
Kruse, Morissette, Young, and Gulliver present a critique of current treatments for post-deployment symptoms, highlighting problems experienced by individuals with blast concussion. A review of this magnitude is necessary to understand the complex nature of treatment for cognitive symptoms in the face of multiple neuropsychiatric concerns and comorbidities.

Lastly, Betthauser, Bahraini, Krengel, and Brenner provide an overview of the literature on self-report measures which have been used routinely as diagnostic and screening tools to document mild traumatic brain injury.

Together, these papers reveal the limitations of current studies which are naturalistic in design and lack experimental control. Confounding factors include multiple medical, social, and cognitive problems arising from numerous etiologies. Further confounds involve time since injury, number of injuries, and symptom trajectories. Although many studies have used similar measures in other contexts, the sensitivity and specificity of these measures in wartime populations have not been fully assessed. And selfreport inventories, although an important part of the diagnostic process, have not been validated for use as stand alone measures in documenting symptomatology for diagnostic purposes and run the risk of missing affected soldiers unaware of their disability.

These reviews are intended to document our current knowledge of illness characteristics in these deployed men and women. It is essential to present a current and thoughtful literature review of the neurocognitive, neuroradiological, and self-report diagnostic tools in addition to interventions available to affected individuals as they continue to need substantial and sustained psychological care and neuro-rehabilitation long after they return from deployment in selfless service to their country. 